\title{
Connecting During the Virtual Interview Process: Lessons from Experience
}

\author{
Lauren D. Feld ${ }^{1}\left[\right.$. Neeral L. Shah ${ }^{2}$
}

Received: 22 October 2020 / Accepted: 20 November 2020 / Published online: 12 January 2021

(c) Springer Science+Business Media, LLC, part of Springer Nature 2021

Due to the COVID-19 pandemic, the annual National Resident Matching Program (NRMP) fellowship match [1] has transitioned to virtual interviews for the current recruitment season [2, 3]. An excellent article by Dr. Mallepally and colleagues outlines recommendations for thoughtful implementation of this major change, describing challenges faced by both applicants and fellowship programs [4]. The authors of this letter had personal experience with virtual interviews in 2018, prior to the pandemic. This experience provided early insight into challenging aspects of virtual interviewing: conveying a program's culture and assessing the intangible quality of "fit."

An important way to determine the mutual fit of a program is to create a meaningful connection between applicants, fellows and faculty. This was possible in the virtual space, but did require creativity. For example, one program arranged for meeting fellows in an informal setting. Another program's coordinator gave the applicant a live virtual department tour by walking with an iPad. To help a diverse set of applicants to visualize themselves in a program, it is important to involve faculty and fellows from different backgrounds. Programs can help applicants envision living in a city by including videos or presentations about the surrounding area. Programs can also build on these resources and convey a sense of inclusivity by describing neighborhoods, affordability, and childcare opportunities.

Many intangible criteria utilized by programs in rank list decisions can work against members of underrepresented

Lauren D. Feld

lfeld@uw.edu

Neeral L. Shah

ns3zt@virginia.edu

1 Department of Gastroenterology, University of Washington, 1959 NE Pacific St., Box 356424, Seattle, WA 98195, USA

2 Division of Gastroenterology and Hepatology, University of Virginia, PO Box 800708, West Complex - GI Offices, Charlottesville, VA 22908, USA groups. Providing anti-bias training for interviewers is helpful [5]. Coordinating and requiring interviewers to use behavioral interviewing questions can reduce implicit bias between applicants. Additionally, program directors have reported that the most important factors when ranking candidates are interview interactions, interpersonal skills, and professionalism [6]. While the article by Dr. Mallepally et al. highlights recommendations on how to demonstrate professionalism in a virtual interview (including attention to an interviewee's physical space, lighting, and noise), program directors must remember that many applicants are now often interviewing in non-traditional circumstances. Applicants may have children around the home who are unable to attend daycare or school. Socioeconomic disparities exist between applicants who can afford quiet, well-lit spaces at home, and those who need to use hospital space. Lack of access to certain resources or spaces should not be construed as indicative of performance during fellowship. Professionalism standards are important, but should not disadvantage certain socioeconomic, cultural, racial, and ethnic groupsconducting interviews through the lens of mitigating bias for underrepresented applicants is essential $[7,8]$. Indeed, a potential benefit to this new virtual process is reducing travel expense inequities as a barrier to enter the field $[9,10]$.

Both applicants and program directors should go through this new virtual interview process with the expectation that "glitches" will occur, and do not necessarily reflect on a program's ability to provide excellent training, or an applicant's ability to be an outstanding fellow. We believe the future of fellowship recruitment will involve some hybrid model of in person and virtual interviews. As this may become the new norm, it is important we continue to refine the process.

Funding The authors have not received grant support or other funding related to this work. 


\section{References}

1. Roth AE. The origins, history, and design of the resident match. JAMA. 2003;289:909-912.

2. Duong N, Aby ES, Hathorn KE, Simons-Linares CR, Bilal M. How to become a competitive applicant for gastroenterology fellowship: tips and tricks for success part I. Dig Dis Sci. 2020;65:1895-1898.

3. Frequently Asked Questions. https://mk0nrmp3oyqui6wqfm.kinst acdn.com/wp-content/uploads/2020/05/NRMP-FAQ-27May2020final.pdf. Accessed 29 June 2020.

4. Mallepally N, Bilal M, Hernandez-Barco YG, Simons M, Berzin TM, Oxentenko AS. The new virtual reality: how COVID-19 will affect the gastroenterology and hepatology fellowship match. Dig Dis Sci. (Epub ahead of print). https://doi.org/10.1007/s1062 0-020-06432-x.

5. Marcelin JR, Siraj DS, Victor R, Kotadia S, Maldonado YA. The impact of unconscious bias in healthcare: how to recognize and mitigate it. J Infect Dis. 2019;220:S62-S73.
6. Release NRMPD, Committee R. Results of the 2016 NRMP program director survey: National resident matching program, data release and research committee; 2016.

7. Nwora C, Allred DB, Verduzco-Gutierrez M. Mitigating bias in virtual interviews for applicants who are underrepresented in medicine. J Natl Med Assoc 2020.

8. AbdelHameid D. Professionalism 101 for black physicians. $N$ Engl J Med 2020.

9. Fogel HA, Liskutin TE, Wu K, Nystrom L, Martin B, Schiff A. The economic burden of residency interviews on applicants. Iowa Orthop J. 2018;38:9-15.

10. Tseng J. How has COVID-19 affected the costs of the surgical fellowship interview process? J Surg Educ 2020.

Publisher's Note Springer Nature remains neutral with regard to jurisdictional claims in published maps and institutional affiliations. 